\section{An inexpensive oculometer for human factors research}

\author{
A. G. GALE \\ Division of Radiology, University Hospital and Medical School \\ Queen's Medical Centre, Nottingham NG7 2UH, England
}

The majority of eye movement recording methods require either attachment to the subject or rigid location of the subject's head. Such constraints are often untenable in many human factors applications. The oculometer technique has none of these drawbacks. Although the fundamental principle of this method is simple, the approach is characterized by high capital expenditure due to the on-line analysis typically employed. The system described here uses manual scoring of the data with subsequent off-line analysis, which keeps the initial outlay at a minimum. The basic oculometer principle is detailed, together with a description of the apparatus and the analysis technique.

The selection of a particular eye movement recording method depends upon several factors, including the nature of the visual task concerned, the recording accuracy required, and the cost. (Refer to Young \& Sheena, 1975, for a recent survey of eye movement recording techniques.) A variant on the corneal reflection theme is the oculometer described by Merchant, Morrissette, and Porterfield (1974), which requires no head attachments. A measurement accuracy of about 1 deg has been reported with this method. The technique typically employs on-line data analysis, which can be expensive.

This paper describes a versatile oculometer in which the data are manually scored, enabling the additional facility of on-line analysis at a later date. By approaching the problem in this manner, it is possible to ascertain future software and hardware needs on the basis of current data trends.

\section{BASIC PRINCIPLE}

Simple corneal reflection methods are extremely sensitive to the effects of head movements (Cowey, 1963). A small head movement can thus be mistaken for an eye movement. This problem can be overcome either by firmly fixing the head (Mackworth, 1967) or by mounting both the light source and the recording device on the head (Mackworth \& Thomas, 1962). An alternative solution is to study two elements of the eye that move differentially only for an eye movement but maintain their relative relationship for a head movement. Two appropriate elements are the observed pupil

This work was supported by a research grant from the Social Science Research Council and was carried out at the Department of Human Sciences, Loughborough University of Technology. center and some suitable corneal reflection (e.g., Gale, 1980; Haith, 1969).

Such a solution is utilized in the oculometer described here. The illumination and recording optics are coaxially aligned, and the relationship between the observed pupil center and the single corneal reflection of the illuminating source forms the basic measurement. Owing to this alignment, the image of the source always appears in line with the center of corneal curvature (at least over the central $25 \mathrm{deg}$ of the cornea that can be considered as approximating a convex mirror). The displacement of the corneal reflection from the observed pupil center is then a function of eye rotation only (Figure 1). The displacement (D) is given by:

$$
\mathrm{D}=\mathrm{K} \times \sin \theta
$$

where $\theta$ is the angle of inclination of the eye's optical axis to the oculometer and $\mathrm{K}$ is the distance from the center of corneal curvature to the observed pupil center. The observed pupil is the entrance pupil, which is the virtual corneal image of the true pupil and lies anterior to it (Bennett \& Francis, 1962). Taking the corneal radius as $7.8 \mathrm{~mm}$ and the distance of the entrance pupil behind the corneal surface as typically $3 \mathrm{~mm}$ (Bullinger, 1974), $\mathrm{K}$ will have a value of some $4.8 \mathrm{~mm}$.

\section{THE OCULOMETER}

The apparatus as used in an industrial inspection situation is shown in Figure 2. A 150-W lamp and two collimating lenses are positioned so as to produce a parallel beam of light limited to the near infrared region by suitable filter (Corning C5-7-69) and incident on the subject's eye. A silicon diode television camera (Shibaden HU 405) fitted with a zoom lens is coaxially aligned with the projection system by means of the half-silvered mirror. Acquisition of the subject's eye is achieved by a rotatable front-silvered mirror fitted in the final common light path. The rest of the recording apparatus is shown in Figure 3; it consists of a video timer (For-A) for noting the time of day and logging other data, a video recorder, and a television monitor. The use of incident infrared illumination has two advantages. First, it produces very little subject awareness of the monitoring process. Second, it aids data analysis, as the coaxial alignment of the illumination and recording optics results in light scattered back from the retina, effectively backlighting the pupil so that it appears as a bright disk (the so-called "bright pupil") distinct from the rest of the eye and facial details, with the corneal reflection of the light source appearing as a much brighter spot. This greatly facilitates the determination of the pupil (Figure 4).

The criterion adopted in setting up the apparatus was 


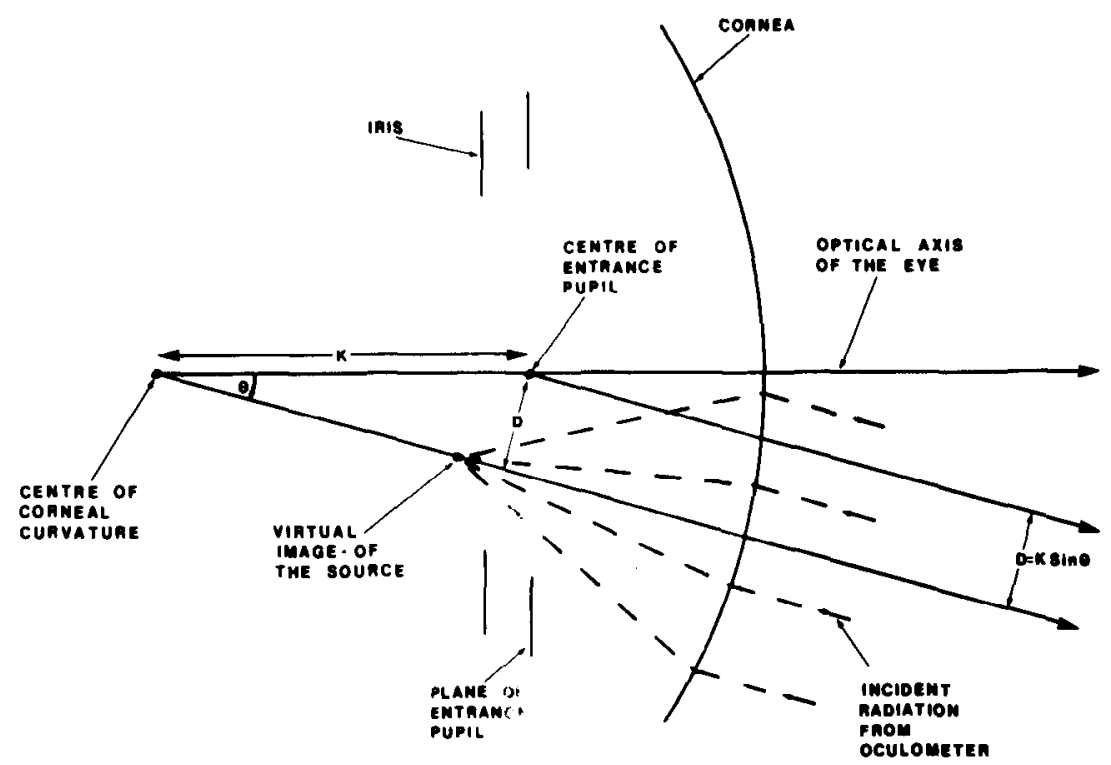

Figure 1. Two-dimensional representation of the ye showing the location of the entrance and true pupils, together with the major ax:s if concem here. A single nodal point is shown for simplicity. Inciden roculometer radiation is reflected by the cornea to form a virtual image of the source. The measurement $\quad$ D) is the discrepancy between this and the center of the entrance pupil as measur' $\mathrm{i}$ " a plane perpendicular to the oculometer alignment.

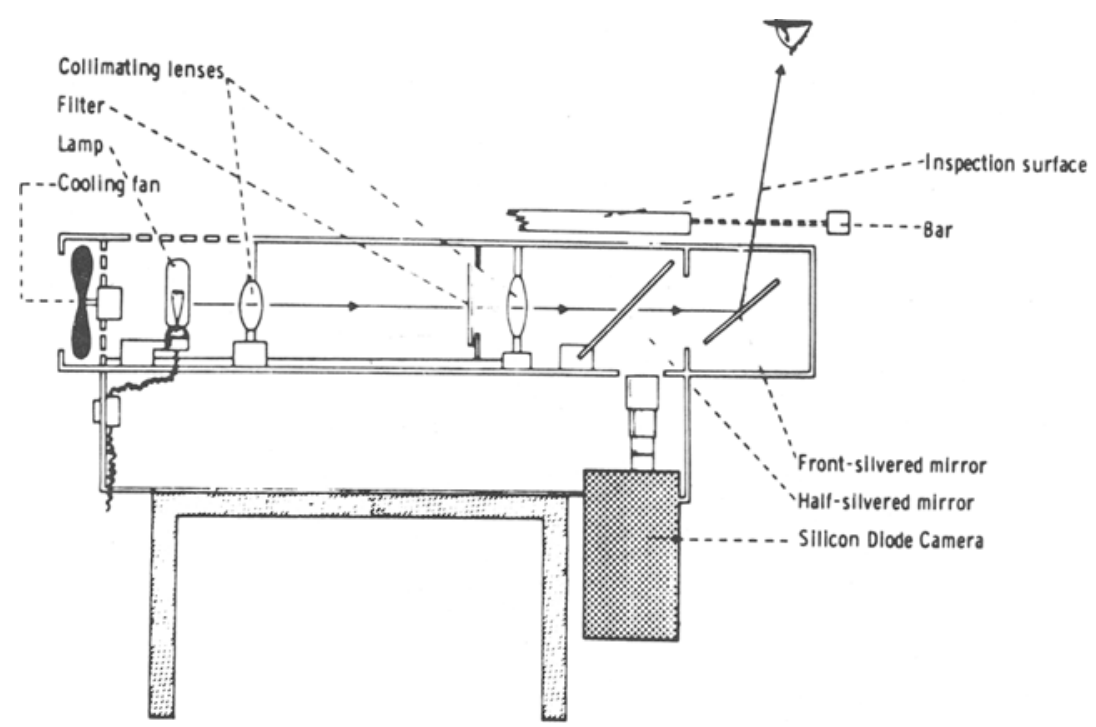

Figure 2. The oculometer mounted beneath an $n$ :pection table. The bar attached to the table prevented accidental damag to the appara $u$

to achieve a usable video picture with the minimal amount of infrared radiation incident on the subect's eye. This was to overcome any possible ocular radiation hazards. The Corning filter passes wavelengths betwen 700 and $1,100 \mathrm{~nm}$. About $40 \%$ of this inciden ocular radiation reaches the retina; the rest is absorbed in the ocular media (Sliney \& Freasier, 1973).

The ocular irradiance was measured over the transmission range of the Corning filter at various distances from the oculometer. The maximum value found was
Ies: than $1 \%$ of the advised limit given by Sliney and F ease e 1973) for long-term chronic exposure to i: frared optical sources $\left(.1 \mathrm{~W} / \mathrm{mm}^{2}\right)$. The retinal irradianc at the typical operating distance from the oculometer was also less than $1 \%$ of the retinal hazard value $\left(1.25 \mathrm{~mW} / \mathrm{mm}^{2}\right.$ ) given by Clark (1968).

In the situation shown in Figure 2, an industrial inspector stood at a work station examining items of Nottingham lace. The oculometer was positioned beneath the inspection table, with the front-silvered 

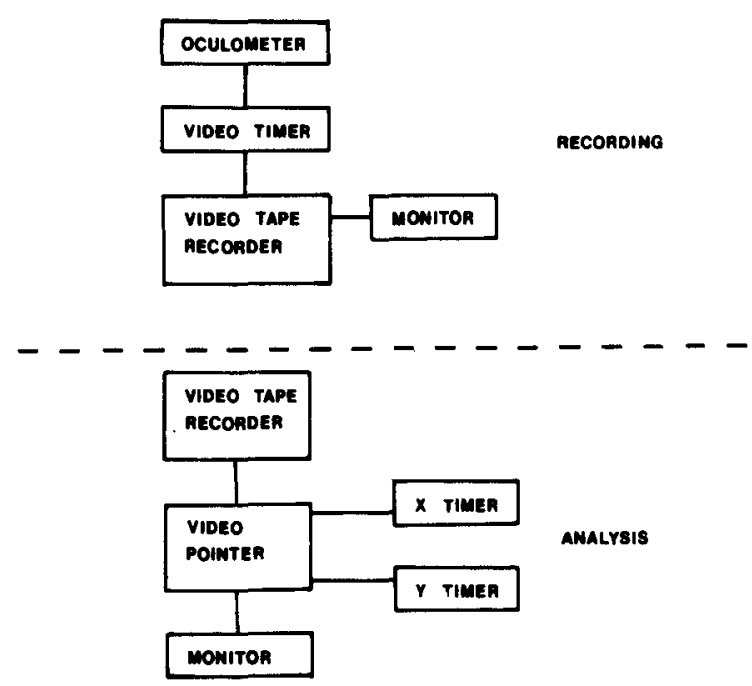

Figure 3. Schematic outline of the apparatus arrangements for the recording and analysis of the data.

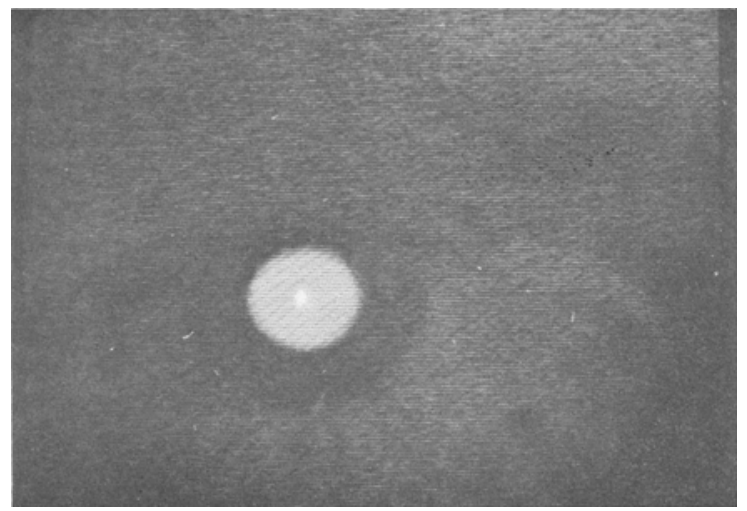

Figure 4. The recorded bright pupil. The comeal reflection of the oculometer light source appears as a bright spot with the backlit pupil as a less bright disk.

mirror projecting some $150 \mathrm{~mm}$ toward the subject. A metal bar attached to the front of the table prevented accidental damage to this mirror. The oculometer was set up by rotating the final mirror until an image of the subject's eye was seen on the monitor. The zoom lens was then adjusted to produce an adequate size of image of this eye on the monitor. The size of eye image is determined by the resolution required, which can be found with Equation 1. Given that $\mathrm{D}$ can be measured to one television line, this becomes $\theta=\left(\sin ^{-1} 1\right) /$ $(\mathrm{K} \times \mathrm{N})$, where $\mathrm{N}$ is the number of television lines per millimeter at the eye.

The size of image utilized is a compromise between the resolution required and the need to allow for some head movements without loss of this image during the inspection of the stimulus material.

In this simple oculometer, the amount of lateral head movement permissible without any loss of the recorded image of the eye is ultimately determined by the diameter of the incident light beam.

Once the apparatus is set up, the experimenter can discreetly monitor the inspector's visual behavior from a distance and can start and stop recording without the subject's knowledge. On calibration trials, each subject is asked to fixate known points on a display placed on the inspection table.

\section{DATA ANALYSIS}

The videotape recording was examined by means of slow-motion playback. In general, every 10 th television frame was analyzed (this being adequate for detecting saccadic movements), unless the data logged on the video picture indicated the need for a modification to this rate. Once a particular frame was selected, the $X$ and $Y$ coordinate positions were taken of points of interest. This was accomplished by adding an arrow (a modified For-A video pointer) to the input video signal (Figure 3). Rapid superimposition of this arrow over a point of interest was accomplished by a joystick and additional fine-position controls. The location of the arrow on the monitor was recorded by two timers. One of these was triggered by the frame count and gave the vertical position. The other timer was triggered by the line pulse and recorded the horizontal position.

For each selected frame, the coordinates of the center of the corneal reflection and of several points around the edge of the pupil were taken. These measures, together with the number of video frames between each set of readings, formed the basic data, which were then analyzed off-line.

To determine the subject's direction of gaze, the center of the pupil was first calculated, using a best-fit procedure applied to the coordinates taken around the pupil edge. The angular disparity of the center of the corneal reflection from the pupil center was then derived, which gave the inclination of the optical axis of the subject's eye to the oculometer axis. The subject's direction of gaze (visual axis) was then finally calculated, using corrections determined from the calibration trials on which subjects fixated known points, and the estimated direction of gaze was compared with each known fixation location. The oculometer as described in this situation had a recording accuracy of $1.5 \mathrm{deg}$.

\section{CONCLUSIONS}

The system described here has produced a simple and inexpensive solution to the problem of discreetly monitoring the visual search behavior of industrial inspectors. Having demonstrated the feasibility of the oculometer approach in this setting, work is now in progress to incorporate a microprocessor to automate the data analysis. 


\section{REFERENCES}

Bennett, A. G., \& Francis, J. L. The eye as an optical system. In H. Davson (Ed.), The eye (Vol. 4). New York: Academic Press, 1962.

Bullinger, A. L'enregistrement des mouvements oculaires par le reflelt corneen. Analyse des erreurs systematiques de la localisation du regard. Perception, 1974, 3, 355-360.

Clark, B. A. J. Welding filters and thermal damage to the retina. Australian Journal of Optometry, 1968, 51, 91-98.

CowEY, A. The basis of a method of perimetry with monkeys. Quarterly Journal of Experimental Psychology, 1963, 15, $81-90$.

GALE, A. G. The role of eye movements in figure perception. Unpublished doctoral thesis, University of Durham, Durham 1980.

HAITH, M. M. Infra-red television recording and measurement of ocular behavior in the human infant. American Psychologist, $1969,24,279-283$.
Mackworth, N. H. A stand camera for line-of-sight recording. Perception \& Psychophysics, 1967, 2, 119-127.

Mackwonth, N. H., \& Thomas, E. L. Head mounted eye-marker camera. Joumal of the Optical Society of America, 1962, 52, 713-716.

Merchant, J., Mornissette, R., \& Ponterfield, J. L. Remote measurement of eye direction allowing subject motion over one cubic foot. IEEE Transactions on Biomedical Engineering, 1974, BME-21, 309-317.

Sliney, D. H., \& Freasier, B. C. Evaluation of optical radiation hazards. Applied Optics, 1973, 12, 1-24.

Young, L., \& Shenna, D. Survey of eye movement recording methods. Behavior Research Methods \& Instrumentation, 1975, 7, 397-429.

(Received for publication March 3, 1981; revision accepted April 24, 1981.) 